\title{
Pro und Kontra: Ist subjektive Lebensqualität ein sinnvoller Outcomeindikator?
}

\author{
For and Against: Is Quality of Life a Meaningful Indicator of Outcome?
}

Pro

Reinhold Kilian

Seit ihrer Entdeckung durch die Psychiatrie in den frühen 80erJahren ist die Lebensqualitätsforschung immer wieder mit enthusiastischen Erwartungen aber auch mit harscher Kritik überzogen worden [1-6]. Trotz einer mehr als 25-jährigen Forschungstradition und trotz der Tatsache, dass der Begriff der Lebensqualität mittlerweile von Psychiatern und Patienten aber auch von der Politik und der pharmazeutischen Industrie als Outcomekriterium psychiatrischer Behandlung akzeptiert ist, besteht unter Wissenschaftlern zum gegenwärtigen Zeitpunkt weder hinsichtlich des Stellenwertes der Lebensqualitätsforschung, noch bezüglich grundlegender methodischer Standards der Erfassung und Analyse von Lebensqualität Übereinstimmung.

Die Ursache dieser Entwicklung ist in der fehlenden theoretischen Reflexion des Lebensqualitätskonzeptes durch die angewandte Forschung [7] und einer daraus resultierenden falschen Erwartung an die Eindeutigkeit und die Aussagekraft einschlägiger Forschungsergebnisse zu sehen. Vor dem Hintergrund dieser These soll versucht werden, den möglichen Nutzen der Lebensqualität für die psychiatrische Versorgungsforschung zu bestimmen und die Bedingungen für die Realisierung dieses Potenzials zu definieren.

\section{Die Entwicklung der Lebensqualitätsforschung in der Psychiatrie}

Um die Entwicklung der Lebensqualitätsforschung in der Psychiatrie zu verstehen, ist es notwendig, sich die Ausgangssituation der psychiatrischen Versorgung in der Mehrzahl der westli- chen Industrieländern zu Beginn der 80er-Jahre des vorigen Jahrhunderts zu vergegenwärtigen. Zu diesem Zeitpunkt war der Enthusiasmus der in den späten 60er-Jahren begonnenen, allgemein mit dem Begriff der Deinstitutionalisierungsbewegung bezeichneten, Bemühungen zur Reform der psychiatrischen Versorgung einer weitgehenden Ernüchterung gewichen $[8,9]$. War zu Beginn dieser Bewegung auch unter Experten die Vorstellung verbreitet, dass die sekundären Begleiterscheinungen schwerer psychischer Erkrankungen, wie soziale und berufliche Desintegration und der Verlust alltagspraktischer Fähigkeiten, eine Folge der totalen Institutionalisierung darstellten, wurde sehr bald deutlich, dass viele der Patienten, auch nach einer Entlassung aus der stationären Versorgung, neben einer medizinischen Behandlung, dauerhaft eine mehr oder weniger intensive Unterstützung in verschiedenen Bereichen der Lebensführung benötigen $[8,10-12]$. Das Fehlen entsprechender gemeindepsychiatrischer Versorgungsangebote führte nicht selten dazu, dass insbesondere ehemalige Langzeitpatienten von psychiatrischen Großkrankenhäusern nach ihrer Entlassung in extreme Notlagen bis hin zur Obdachlosigkeit gerieten $[9,10,13,14]$. Eine Reihe von Autoren vertraten damals die These, dass die klassischen Instrumentarien zur Beurteilung der Wirksamkeit psychiatrischer Behandlung nicht mehr ausreichten, um den Anforderungen gerecht zu werden, die sich aus dem Anspruch ergaben, Menschen mit schweren chronischen psychischen Erkrankungen ein Leben außerhalb von Anstaltsmauern zu ermöglichen [8,14-19]. Da sich eine so verstandene psychiatrische Versorgung zwangsläufig auf alle relevanten Lebensbereiche der Patienten erstrecken musste, wurde zur Beurteilung der Effektivität der Versorgung ein Instrumentarium benötigt, welches dieses breite Behandlungsspektrum adäquat erfassen konnte. Mit dem wenige Jahre früher im Rahmen der Sozialindikatorenforschung entwickelten Fragebogen zur subjektiven Lebensqualität $[20,21]$ glaubte man damals ein derartiges Instrumentarium gefunden zu haben, dass lediglich noch einiger Modifizierungen für den Bereich der psy- 
chiatrischen Versorgung bedurfte $[16,18,19]$. Vor dem Hintergrund extrem einfacher theoretischer Modelle [22] wurde damals selbstverständlich angenommen, subjektive Lebensqualität sei messbar und sie könne durch therapeutische Interventionen oder durch Veränderung objektiver Lebensbedingungen beeinflusst werden [15,23-25].

Im Verlauf der 80er-Jahre wurden dann eine Reihe von Fragebögen zur Erfassung der Lebensqualität von Menschen mit schweren psychischen Erkrankungen entwickelt und in einer Vielzahl von Studien eingesetzt $[4,26]$. Verschiedene Bestandsaufnahmen der Ergebnisse dieser Studien kamen einhellig zu der Schlussfolgerung, dass die gefundenen Ergebnisse mit den, den verwendeten Erhebungsverfahren zugrunde liegenden einfachen theoretischen Annahmen nicht zu vereinbaren waren [1,2,4,27-31]. Als Konsequenz dieser Einsicht wurden verschiedene theoretische Modelle entwickelt, die versuchten, der Komplexität des Verhältnisses zwischen Mensch und Umwelt unter der besonderen Bedingung psychischer Erkrankung gerecht zu werden $[4,27]$.

Ebenso wie bereits verschiedene Autoren im Bereich der allgemeinen Lebensqualitätsforschung [32 - 35] kamen Kilian [1] sowie Angermeyer und Kilian [4] im Rahmen dieser Bemühungen zu dem Ergebnis, dass die subjektive Lebensqualität nicht als Ergebnis eines rekursiven Beurteilungsprozesses betrachtet werden kann, sondern dass vielmehr die menschliche Fähigkeit, eigene Ansprüche und Erwartungen an wahrgenommene Gegebenheiten anzupassen, eine zentrale Rolle spielen. Subjektive Lebensqualität wird in diesem Modell nicht als Ergebnis eines rekursiven Prozesses, sondern, wie z.B. die Körpertemperatur, als Regelgröße innerhalb eines kybernetischen Mensch-UmweltSystems betrachtet. Gestützt wird diese theoretische Perspektive durch Arbeiten von Robert Cummins, der die Annahme vertritt, dass Menschen ein bestimmtes, universelles Niveau des subjektiven Wohlbefindens aufweisen, dessen intra- aber auch interpersonelle Varianz sehr gering ist [32 - 34]. Vor dem Hintergrund einer Vielzahl von Studien liegt dieses Niveau nach Cummins auf einer Skala von 0 - 100 ungefähr bei 75 [34].

Durch ihre immense Adaptationsfähigkeit sind Menschen in der Lage, dieses Niveau subjektiven Wohlbefindens unter variierenden Umweltbedingungen aufrechtzuerhalten. Voraussetzungen dieser menschlichen Adaptationsfähigkeit sind nach Cummins et al. bestimmte Persönlichkeitsmerkmale, die allgemein als die „big five“ bezeichnet werden und die im Prozess der MenschUmwelt-Interaktion Pufferfunktionen ausüben, welche insbesondere die potenzielle negative Wirkung aversiver Umweltbedingungen auf das subjektive Wohlbefinden abfedern [34]. Die Ausprägungen dieser Persönlichkeitsmerkmale haben nach Cummins et al. einen wesentlichen Einfluss auf die Stabilität der Wohlbefindenshomöostase. Allerdings wird dieser Zusammenhang dadurch erheblich kompliziert, dass die Bedeutung der Persönlichkeitsmerkmale mit den jeweiligen Umweltbedingungen interagiert. D.h. die Pufferwirkung bestimmter Ausprägungen von Neurotizismus, Extraversion und anderer Persönlichkeitsmerkmale hängt wiederum vom Grad der Aversivität der Umweltbedingungen ab. Neben Persönlichkeitsmerkmalen wurden auch eine Reihe von kognitiven Orientierungsmustern, wie z.B. Kontrollüberzeugungen, Selbstbewusstsein und Optimismus als
Einflussfaktoren der Wohlbefindenshomöostase identifiziert $[33,34]$. Auch für diese kognitiven Faktoren gilt, dass ihr Einfluss nicht rekursiv und linear ist, sondern ebenfalls mit den jeweiligen Umweltbedingungen variiert [33].

Ohne an dieser Stelle die komplexen Zusammenhänge zwischen Wohlbefindenshomöostase, Persönlichkeitsmerkmalen und Umweltbedingungen weiter ausführen zu können, soll versucht werden, die Implikationen der dargestellten theoretischen Überlegungen für die Frage der Eignung des Konstruktes der subjektiven Lebensqualität als Indikator für die Beurteilung der Qualität der psychiatrischen Versorgung herauszuarbeiten.

\section{Implikationen eines homöostatischen Lebensqualitäts- konstruktes für die psychiatrische Versorgungsforschung}

Die Betrachtung von subjektiver Lebensqualität als Regelgröße eines homöostatischen Prozesses impliziert, dass kein rekursiver Zusammenhang zwischen subjektiver Lebensqualität und Umweltbedingungen besteht. Allerdings zeigen die obigen Ausführungen, dass der Prozess der Wohlbefindenshomöostase keineswegs unbeeinflusst von Umweltbedingungen abläuft. Bleibt man bei der Analogie zur Körpertemperatur, so könnte man vermuten, dass, obwohl niemand auf die Idee käme von der Körpertemperatur eines Menschen direkt auf die Temperatur der Luft oder die Qualität der Kleidung zu schließen, bei einer extremen Abweichung der Körpertemperatur vom Normalwert das Vorliegen aversiver Umweltbedingungen als eine mögliche Ursache in Betracht gezogen würde. Gleichzeitig wird die Tatsache, dass die Körpertemperatur eines Menschen normal ist, zwar häufig als ein Indikator körperlicher Gesundheit herangezogen, kaum jemand glaubt jedoch, dass die Gesundheit eines Menschen ausschließlich über die Messung der Körpertemperatur beurteilt werden kann. Betrachtet man subjektive Lebensqualität aus diesem Blickwinkel, so lassen sich einige Aussagen darüber formulieren, in welcher Weise diese als Indikator für Versorgungsqualität in der Psychiatrie verwendet werden kann.

Zum einen lässt sich feststellen, dass immer dann, wenn die subjektive Lebensqualität eines Menschen mit einer psychischen Erkrankung gegenüber der der Allgemeinbevölkerung schlechter ist, ein Versorgungsdefizit vorliegt. Dies gilt auch dann, wenn die Beurteilung der subjektiven Lebensqualität durch psychopathologische Symptome, z.B. Depressivität oder Negativsymptome beeinflusst wird. Die Behandlung der Depressivität oder der Negativsymptomatik ist Aufgabe der psychiatrischen Versorgung und ein negativer Einfluss dieser Symptome auf die subjektive Lebensqualität signalisiert eine fehlende Behandlungseffektivität.

Eine subjektive Lebensqualität auf dem Niveau der Allgemeinbevölkerung oder darüber kann demgegenüber nicht als hinreichender Indikator für eine gute Versorgungsqualität angesehen werden, da sich darin eine resignative Anpassung des Aspirationsniveaus an deprivierte Lebensbedingungen widerspiegeln kann. Eine derartige Form der Anpassung muss immer dann in Betracht gezogen werden, wenn ein hohes Niveau der subjektiven Lebensqualität mit schlechten objektiven Lebensbedingungen einhergeht. 
Eine Verbesserung der subjektiven Lebensqualität, z.B. als Folge einer psychiatrischen Intervention, kann nur dann erwartet werden, wenn das Ausgangsniveau deutlich unter dem der Allgemeinbevölkerung liegt oder wenn durch die Intervention objektive Umweltbedingungen geschaffen werden, die deutlich besser sind als die der Allgemeinbevölkerung. Da die subjektive Lebensqualität psychiatrischer Patienten als Folge resignativer Anpassung häufig ähnlich hoch ist wie in der Allgemeinbevölkerung und da die Effekte psychiatrischer Interventionen auf die Lebensbedingungen der Patienten in der Regel sehr gering sind, kann nur in seltenen Fällen mit einem statistisch signifikanten Effekt einer psychiatrischen Intervention auf die subjektive Lebensqualität der Patienten gerechnet werden.

In Querschnittstudien ist das Fehlen von Zusammenhängen zwischen objektiven Lebensbedingungen und subjektiver Lebensqualität völlig plausibel, da hier lediglich das Ergebnis eines Anpassungsprozesses erfasst werden kann. Um unter, gemessen am jeweiligen soziokulturellen Standard, normalen Umständen derartige Zusammenhänge erfassen zu können, sind grundsätzlich Längsschnittstudien notwendig. Als Beispiel siehe Kilian et al. 2005 [36], die zeigen, dass eine Veränderung objektiver Lebensbedingungen tatsächlich zu einer Veränderung der subjektiven Lebensqualität führt, wenn tatsächlich die Veränderungen und nicht die korrelativen Zusammenhänge im Querschnitt gemessen und analysiert werden.

Allerdings kann auch bei der Anwendung elaborierter Analysemethoden nicht erwartet werden, dass sich die subjektive Lebensqualität psychiatrischer Patienten langfristig verbessert. Im Gegenteil, bei einer im Zusammenhang mit einer dauerhaft wirksamen Intervention zeitnahen längsschnittlichen Messung der subjektiven Lebensqualität ist nach einer kurzfristigen Verbesserung sogar mit einer Verschlechterung der subjektiven Lebensqualität zu rechnen, da sich aufgrund des Erlebens besserer Umweltbedingungen bei den Patienten eine Steigerung des Aspirationsniveaus einstellen kann. Da eine derartige Erhöhung des Aspirationsniveaus aus therapeutischer Sicht durchaus wünschenswert ist, könnte eine Verschlechterung der subjektiven Lebensqualität unter Umständen eine gute Versorgungsqualität indizieren.

Vor- und Nachteile eines derartigen Ergebnisindikators liegen auf der Hand. Subjektive Lebensqualität kann in der Regel als Indikator einer schlechten Versorgungsqualität, nicht jedoch als Indikator einer guten Versorgungsqualität herangezogen werden. Zur Interpretation der subjektiven Lebensqualität psychiatrischer Patienten werden Referenzwerte für die Allgemeinbevölkerung benötigt. Die Entdeckung von Zusammenhängen zwischen der Veränderung objektiver Lebensbedingungen und subjektiver Lebensqualität erfordert aufwändige Längsschnittstudien und entsprechend elaborierte Analyseverfahren. Eine Veränderung der subjektiven Lebensqualität lässt sich darüber hinaus nur im Zusammenhang mit Informationen über gleichzeitige Veränderungen des Aspirationsniveaus sinnvoll interpretieren.

Die Verwendung der subjektiven Lebensqualität als Ergebniskriterium der psychiatrischen Versorgungsforschung ist demzufolge sinnvoll, wenn das Ziel dieser Forschung darin besteht, die De- fizite bestehender Behandlungs- und Hilfsangebote aufzudecken und Hinweise auf die Möglichkeiten zur Behebung dieser Defizite zu gewinnen. Keinerlei Sinn macht die Verwendung subjektiver Lebensqualität dagegen in Studien, die ausschließlich darauf abzielen, eine gute Qualität oder gar Effektivität psychiatrischer Interventionen nachzuweisen. Sieht man die vorrangige Aufgabe der Wissenschaft in der Erzeugung von Gewissheiten, so mag man diese Tatsache bedauern, sieht man die Aufgabe von Wissenschaft dagegen in der Aufdeckung von Irrtümern und der Enttäuschung falscher Erwartungen, so kann man ihr gefasst ins Auge sehen.

Dr. rer. soc. Reinhold Kilian

Arbeitsgruppe Versorgungsforschung

der Klinik für Psychiatrie II

der Universität Ulm

Ludwig-Heilmeyer-Straße 2

89312 Günzburg

E-mail: Reinhold.Kilian@bkh-guenzburg.de

\section{Kontra}

\section{Ralf Pukrop}

Der für die somatischen Fächer maßgebliche ideologische Hintergrund für die Einführung des Lebensqualitäts(LQ)-Konzeptes besteht in einer stärkeren Beachtung einer vormals weitgehend ausgeblendeten, subjektiven Patientenperspektive. Diese zentrale Legitimationsquelle existiert in diesem Sinne für den psychiatrischen Kontext kaum, da hier das Subjektive ohnehin einen immanenten Bestandteil des Fachgebietes definiert. Psychische, körperliche und soziale Dimensionen als die wichtigsten Teilbereiche der LQ sind bereits Bestandteil jeder systematisch durchgeführten psychiatrischen Anamnese. Bezieht man darüber hinaus das gesamte Spektrum verfügbarer psychometrisch evaluierter Instrumente mit ein, so ergibt sich ein umfangreicher Bestand an reliablen, validen, sensitiven und normierten Skalen, die theoretisch hinreichend verankerte und hoch differenzierte Konstrukte des subjektiv zugänglichen Variablenkosmos erfassen können. Es bedarf eingehender Begründung, inwieweit ein schlecht definiertes Globalkonstrukt LQ über die bereits verfügbaren spezifischen Indikatoren der subjektiven Erlebniswelt noch hinausgehen könnte.

Im Folgenden sollen die an anderer Stelle [2] ausführlicher behandelten Argumente für einen kritischeren Umgang mit dem LQ-Konstrukt in Form kurzer Hypothesen gelistet werden. Um die aktuelle Gültigkeit dieser Hypothesen zu prüfen, wurde außerdem eine Literaturrecherche durchgeführt, die sich inhaltlich auf LQ und Schizophrenie sowie zeitlich auf die aktuelle Spanne von Januar ' 05 bis Mai '06 beschränkt. Diese Recherche ergab 113 Originalarbeiten ${ }^{1}$ und stützt im Wesentlichen die folgenden Hypothesen:

1. LQ ist nicht hinreichend präzise definierbar. Die inhaltliche Breite und die unscharfen Grenzen des Konstruktes erlauben keine

\footnotetext{
${ }^{1}$ Die Literaturliste kann beim Autor angefordert werden.
} 
präzise Nominaldefinition. Operationale Definitionen scheitern an der Vielzahl der Operationalisierungen (etwa 1000 Messinstrumente implizieren ebenso viele Konstrukte). Für alle empirisch ausgerichteten Wissenschaften stellt eine Definition jedoch die Grundlage wissenschaftlicher Analysen dar.

2. Für die Operationalisierung von LQ existieren keine verbindlichen Kriterien. Selbst wenn man die enge inhaltliche und zeitliche Beschränkung auf das Gebiet der Schizophrenie 2005/06 vornimmt, finden sich in den 113 untersuchten Publikationen 38 verschiedene LQ-Erhebungsinstrumente. Interessanterweise nimmt die Quality of Life Scale (QLS) dabei einen Spitzenplatz ein (21 Studien). Die QLS ist das einzige Fremdbeurteilungsinstrument unter den 38 identifizierten Skalen, sodass hiermit noch nicht einmal der besondere subjektive Charakter der LQ repräsentiert wird.

Des Weiteren können mindestens 50\% der verwendeten Instrumente als generisch klassifiziert werden, da sie nicht spezifisch für den psychiatrischen Kontext erstellt wurden. Aber auch so genannte störungsspezifische Instrumente (wie z.B. das Lancashire Quality of Life Profile und seine diversen Varianten) verdienen eine solche Bezeichnung kaum, da sie ausschließlich LQ-Bereiche erfassen, die sich auch in generischen Instrumenten wiederfinden.

3. LQ besitzt eine unzureichende divergente Validität. Hinter diesem technischen Terminus verbirgt sich die zentrale Schwäche des Konstruktes, da LQ mit einer großen Zahl psychosozialer Merkmale substanziell korreliert ist, und darüber eine eigene Identität kaum feststellbar ist. Die oben definierte Auswahl an Studien liefert dazu eindrückliche Belege: Copingstile, Selbstwirksamkeit, Persönlichkeitsmerkmale, psychopathologische Merkmale, Stresserleben, Nebenwirkungen, soziale Unterstützung u.a. können je nach Berücksichtigung in einer einzelnen Studie den Großteil valider LQ-Varianz aufklären. Drei Merkmale sind besonders hervorzuheben: a) depressiv-ängstliches Syndrom: in mindestens 13 Studien finden sich eindeutige Belege für die Kopplung geringer subjektiver LQ an depressive und ängstliche Symptome. Bei Patienten aus dem psychiatrischen, neurologischen oder allgemeinärztlichen Kontext sowie bei gesunden Kontrollen erklären derartige Symptome 39-73\% der LQ und stellen jeweils die stärkste Determinante dar. Damit sind LQ und Depressivität zwar nicht identisch, es stellt sich jedoch die zentrale Frage, ob depressive Zustände, die eine negative Sicht auf viele Lebensbereiche implizieren, nicht den Kern von LQ-Messungen ausmachen; b) Persönlichkeitsmerkmale: hohe Ausprägungen im Neurotizismus (oder Harm Avoidance) und geringe Ausprägungen auf Extraversion prädisponieren zu einer geringeren LQ; c) die aktuelle Stimmung bei der Beantwortung eines LQ-Fragebogens, der sich eigentlich auf die letzten 2-4 Wochen beziehen soll. Insbesondere klinische Studien zur Prüfung eines pharmakologischen Effektes auf die LQ prüfen nicht hinreichend, inwieweit die LQ-Veränderungen durch die genannten oder verwandte Merkmale bedingt und damit als epiphänomenal zu charakterisieren sind.

4. LQ-Forschung ist nicht hypothesengeleitet. Da LQ-Instrumente fast immer mehrdimensional sind, existiert i.d.R. ein großer Pool potenziell testbarer Hypothesen. Keine der selektierten Stu- dien legt allerdings a priori fest, in welchen LQ-Bereichen aus welchen Gründen Unterschiede erwartet werden, und in welchen Bereichen sich die untersuchten Gruppen nicht unterscheiden sollten. Insbesondere in pharmakologischen Untersuchungen werden nachträglich als signifikant erkannte LQ-Unterschiede zwischen Verum- und Plazebo- oder zwischen zwei mit unterschiedlichen Psychopharmaka behandelten Gruppen berichtet, ohne die große Anzahl der Hypothesen bei der Diskussion zu berücksichtigen. Noch unkontrollierter gestalten sich korrelative Analysen: Hier entstehen schnell große Datenmatrizen, wenn z.B. 10 LQ-Variablen mit 10 weiteren erhobenen Merkmalen korreliert werden. Das nachträgliche Herausfischen der aus dem Gesamtpool potenzieller Hypothesen erhaltenen signifikanten Ergebnisse ist eine Regel, die (fast) ohne Ausnahme bleibt.

Aufgrund der geringen divergenten Validität bzw. der inhaltlichen Breite von LQ erhöht sich zwangsläufig die Wahrscheinlichkeit, mit diesem Konstrukt Veränderungen in irgendeinem psychosozialen Lebensbereich nach einer Intervention innerhalb einer Gruppe oder Unterschiede zwischen verschiedenen Gruppen abzubilden. Darüber hinaus sind etliche Zusammenhänge mit anderen Merkmalen garantiert, die sich dann als „Ergebnis“ einer wissenschaftlichen Untersuchung darstellen lassen.

5. Es gibt keine empirisch prüfbare Theorie zur $L Q$. Über $90 \%$ der selektierten Studien nehmen keinerlei expliziten Bezug zu einem theoretischen Modell oder machen allenfalls vage Andeutungen. Die wenigen Studien, die einen entsprechenden Versuch unternehmen, präsentieren heuristische Modelle, die in erster Linie die vielfältigen Beziehungen des psychosozialen Megakonstruktes LQ zu anderen Variablenklassen widerspiegeln. Die differenziertesten Ansätze postulieren nicht weniger als 30 Variablen, die als Determinanten oder Folgen von LQ in Erscheinung treten. Mit LQ lässt sich (fast) alles prädizieren, und LQ kann durch (fast) alles prädiziert werden. Es handelt sich daher um ein empirisch weitgehend fruchtloses Konstrukt.

Trotz dieser kritischen Haltung möchte ich betonen, dass eine stärkere Berücksichtigung der Patientenperspektive weiter zu unterstützen und auszubauen ist. Angesichts der genannten Schwierigkeiten plädiere ich allerdings für eine wissenschaftlich wesentlich strengere Bewertung, damit LQ nicht zu einem beliebten, da politisch korrekten, aber weitgehend unreflektiert verwendeten Konzept degeneriert. Vor allem ist stets zu überlegen, ob die subjektive Seite nicht durch spezifischere Zielgrößen mit theoretisch besser validierten Konstrukten abgebildet werden kann (z.B. soziale Funktionalität/Adaptation, Selbstwirksamkeit, Symptome, Beschwerden etc.). Insbesondere muss die kumulierte Evidenz über die Bedeutsamkeit depressiver Verstimmungen für eine subjektive Bewertung des eigenen Lebens (wissenschafts-)theoretisch und klinisch deutlich stärker beachtet werden. Wird aber dennoch auf das LQ-Konstrukt zurückgegriffen, so sollten einige Standards mit größerer Verbindlichkeit erfüllt werden, wie sie z.B. von Franz in seinem Übersichtsartikel in diesem Band diskutiert werden. Denn keine der hier näher untersuchten und aufgrund ihrer Aktualität am ehesten den Stand der Wissenschaft repräsentierenden Studien widerlegt die oben aufgestellten Hypothesen in hinreichender Weise (explizite Definition des LQ-Begriffes und begründete Auswahl des Erhebungsinstrumentes, Kontrolle assoziierter psychosozialer Merkmale 
mit einem Schwerpunkt auf ängstlich-depressive Syndrome, Persönlichkeit und aktuelle Stimmungen, a-priori-Formulierung spezifischer Zusammenhangs- oder Unterschiedshypothesen über alle verwendeten LQ-Variablen mit anschließenden statistischen Korrekturen, Einbettung der Ergebnisse in einen theoretischen Kontext).

\section{PD Dr. Dr. Ralf Pukrop}

Klinik und Poliklinik für Psychiatrie und Psychotherapie Klinikum der Universität zu Köln

Kerpener Straße 62

50924 Köln

E-mail: ralf.pukrop@uk-koeln.de

\section{Literatur}

${ }^{1}$ Kilian R. Ist Lebensqualität messbar? Probleme der quantitativen und Möglichkeiten der qualitativen Erfassung von Lebensqualität in der Psychiatrie. Psychiat Prax 1995; 22: $97-101$

2 Pukrop R. Subjektive Lebensqualität. Kritische Betrachtung eines modernen Konstrukts. Nervenarzt 2002; 74: 48 - 54

${ }^{3}$ Katschnig H. How useful is the concept of quality of life in psychiatry? Cur Opinion Psychiatry 1997; 10: 337-345

${ }^{4}$ Angermeyer MC, Kilian R. Theoretical models of quality of life for mental disorders. In: Katschnig H, Freeman H, Sartorius N (eds): Quality of life in mental disorders. Second Edition. Chichester: John Wiley \& Sons, 2006: 21 - 32

${ }^{5}$ Meyer T. Vorstellungen schizophrener Menschen über Lebensqualität. Hamburg: Verlag Dr. Kovac, 2004

${ }^{6}$ Nieuwenhuizen C von. Quality of life of persons with severe mental illness: an instrument. Amsterdam: Thesis Publishers Amsterdam, 1998

${ }^{7}$ Kilian R, Angermeyer MC. Quality of life in psychiatry as an ethical duty: From the clinical to the societal perspective. Psychopathology 1999; 32: 127-134

${ }^{8}$ Lamb HR. Treating the long-term mentally ill. San Francisco: JosseyBass, 1982

${ }^{9}$ Scheper-Hughes N. Dilemmas in deinstitutionalization. A view from inner city Boston. Journal of Operational Psychiatry 1981; 12: 90-99

${ }^{10}$ Bachrach LL. Deinstitutionalisation: promises, problems and prospects. In: Knudsen HC, Thornicroft G (eds): Mental health service evaluation. Cambridge: Cambridge University Press, 1996: 3-18

${ }^{11}$ Häfner $\mathrm{H}$, Heiden W an der. Effectiveness and cost of community care for schizophrenic patients. Hosp Commun Psychiatr 1989; 40: 59-63

12 Bauer M, Kunze H, Cranach M von, Fritze J, Becker T. Psychiatric reform in Germany. Acta Psychiatr Scand 2001; 104 (Suppl 410): 27-34

${ }^{13}$ Estroff SE. Making it crazy. An ethnography of psychiatric clients in an American community. Berkeley: University of California Press, 1981

${ }^{14}$ Lamb HR. What did we really expect from deinstitutionalization? Hosp Commun Psychiatr 1981; 32: 105-109

${ }^{15}$ Malm U, May PRA, Dencker SJ. Evaluation of the quality of life of the schizophrenic outpatient. A checklist. Schizopr Bull 1981; 7: 477-486

${ }^{16}$ Lehman AF. A quality of life interview for the chronically mentally ill. Ev Prog Planning 1988; 11: $51-62$
${ }^{17}$ Lehman AF, Ward NC, Linn LS. Chronic mental patients: The quality of life issue. Am J Psychiatr 1982; 139: 1271 - 1276

18 Bigelow DA, Brodsky G, Stewart L, Olson MM. The concept of quality of life as a dependent variable in evaluation of mental health services. In: Tash W, Stahler G (eds): Innovative approaches to mental health evaluation. New York: New York Academic Press, 1982: 345-366

${ }^{19}$ Baker F, Intagliata J. Quality of life in the evaluation of community support systems. Ev Prog Planning 1982; 5: 69-79

${ }^{20}$ Andrews FM, Withey SB. Social Indicators of well-being. America's perception of life quality. New York: Plenum Press, 1976

21 Campbell A, Converse PE, Rodgers WL. The Quality of American life. New York: Russel Sage, 1976

22 Angermeyer MC, Kilian R. Theoretical models of quality of life for mental disorders. In: Katschnig H, Freeman H, Sartorius N (eds): Quality of life in mental disorders. Chichester: John Wiley \& Sons, 1997: $19-30$

${ }^{23}$ Lehman AF. The well-being of chronic mental patients. Assessing their quality of life. Arch Gen Psychiatry 1983; 40: 369-373

${ }^{24}$ Becker M, Diamond R, Sainfort F. A new patient focused index for measuring quality of life in persons with severe and persistent mental illness. Qual Life Res 1993; 2: 239-251

${ }^{25}$ Herrman H, Hawthorne G, Thomas R. Quality of life assessments in people living with psychosis. Soc Psychiatry Psychiatr Epidemiol 2002; 37: $510-518$

${ }^{26}$ Lehman A. Instruments for measuring quality of life in mental disorders. I: up to 1996. In: Katschnig H, Freeman H, Sartorius N (eds): Quality of life in mental disorders. Chicester: Wiley \& Sons, 2006: $71-84$

${ }^{27}$ Zissi A, Barry MM, Chochrane R. A mediational model of quality of life for individuals with severe mental health problems. Psychol Med 1998; 28: 1221 - 1230

${ }^{28}$ Barry MM, Zissi A. Quality of life as an outcome measure in evaluating mental health services: a review of the empirical evidence. Soc Psychiatry Psychiatr Epidemiol 1997; 32: 38 - 47

${ }^{29}$ Franz M, Meyer T, Reber T, Gallhofer B. The importance of social comparisons for high levels of subjective quality of life in chronic schizophrenic patients. Qual Life Res 2000; 9: 481-489

${ }^{30}$ Franz M, Meyer T, Spitznagel A, Schmidth H, Wening K, Gallhofer B. Responsiveness of subjective quality of life assessment in schizophrenic patients: a quasi-experimental pilot study. Eur Psychiatr 2001; 16: 99-103

${ }^{31}$ Franz M, Meyer T, Gallhofer B. Are importance ratings useful in the assessment of subjective quality of life in schizophrenic patients? Int J Meth Psychiatr Res 1999; 8: 204-211

${ }^{32}$ Cummins RA. Objective and subjective quality of life: An interactive model. Social Indicators Research 2000; 52: 55-72

${ }^{33}$ Cummins RA, Nistico H. Maintaining life satisfaction: The role of positive cognitive bias. Journal of Happiness Studies 2002; 3: 37-69

${ }^{34}$ Cummins RA, Gullone E, Lau ALD. A model of subjective well-being homeostasis: The role of personality. In: Gullone E, Cummins RA (eds): The universality of subjective well-being indicators: A multidisciplinary and multi-national perspective. New York: Springer, 2002: 7-46

35 Diener E, Diener C. Most people are happy. Psychological Science 1996; 7: $181-185$

${ }^{36}$ Kilian R, Angermeyer MC. The effects of antipsychotic treatment on quality of life of schizophrenic patients under naturalistic treatment conditions: An application of random effect regression models and propensity scores in an observational prospective trial. Qual Life Res 2005; $14: 1275-1289$ 